\title{
Revista Colombiana de

\section{La pulsioximetría como herramienta para la tamización de cardiopatías congénitas críticas. Una revisión} narrativa

\section{Melissa Ramírez-Escobar, Jefferson Betancurt-Serrano, Julián Ramírez-Cheyne*, Javier Torres-Muñoz y Antonio J. Madrid-Pinilla}

\author{
Universidad del Valle, Cali, Colombia
}

Recibido el 21 de febrero de 2017; aceptado el 6 de agosto de 2018

Disponible en Internet el 10 de febrero de 2019

\author{
PALABRAS CLAVE \\ Anomalías \\ congénitas; \\ Cardiopatías \\ congénitas; \\ Oximetría; \\ Tamizaje neonatal
}

\begin{abstract}
Resumen
Objetivo: Revisar el concepto de cardiopatía congénita crítica, describir el proceso de consolidación de la pulsioximetría como herramienta para su tamización y llamar la atención sobre la necesidad de implementarla en países de bajos ingresos como Colombia.

Métodos: Se hizo una búsqueda en las bases de datos PubMed-MEDLINE y Scholar Google, usando los términos MeSH: "CongenitalAbnormalities", "HeartDefects, Congenital" "Oximetry" y "Neonatal Screening", cruzando el término "HeartDefects, Congenital" con los demás, por medio del operador booleano AND. Se incluyeron publicaciones desde 1992 y no se excluyeron artículos por metodología o idioma.

Resultados: El $25 \%$ de los casos de recién nacidos vivos con cardiopatía congénita son críticos y la pulsioximetría es una alternativa efectiva para su reconocimiento temprano. Se hizo una revisión sobre el tema, mencionando los estudios con los mayores tamaños de muestra que han permitido la consolidación de la pulsioximetría como herramienta para tamización de cardiopatías congénitas críticas, describiendo el abordaje estadounidense al respecto y llamando la atención sobre la necesidad de implementar dicha tamización en Colombia.

Conclusiones: Existe evidencia para la introducción de la pulsioximetría como prueba de tamización para defectos cardiacos congénitos críticos. En Colombia no se han presentado iniciativas oficiales para generalizar la práctica.

(c) 2018 Sociedad Colombiana de Cardiología y Cirugía Cardiovascular. Publicado por Elsevier España, S.L.U. Este es un artículo Open Access bajo la licencia CC BY-NC-ND (http:// creativecommons.org/licenses/by-nc-nd/4.0/).
\end{abstract}

\footnotetext{
* Autor para correspondencia.

Correo electrónico: julian.andres.ramirez@correounivalle.edu.co (J. Ramírez-Cheyne).
} 


\section{KEYWORDS}

Congenital anomalies;

Congenital heart

diseases;

Oximetry;

Neonatal screening
Pulse oximetry as a screening tool for critical congenital heart diseases. A narrative review

\begin{abstract}
Objective: This article sets out to review the concept of critical congenital heart diseases, as well as to describe the consolidation process with pulse oximetry as a tool for their screening, and to call attention to the need to implement this in low income countries like Colombia.

Methods: A search was made in the PubMed-MEDLINE and Google Scholar databases using the MeSH terms: "Congenital Abnormalities"', "Heart Defects, Congenital" "Oximetry" and "Neonatal Screening", crossing the term "Heart Defects, Congenital" with the rest, by means of the Boolean operator AND. Publications since 1992 were included and article were not excluded due to methodology or language.

Results: Around one-quarter $(25 \%)$ of live newborns with a congenital heart disease are critical, and pulse oximetry is an effective alternative for its early recognition. A review was carried out on the subject, mentioning studies with large sample sizes that have allowed the consolidation of pulse oximetry as screening tool for critical congenital heart diseases. The approach used in the USA in this respect is described, as well as calling attention to the need to implement this screening method in Colombia.

Conclusions: There is evidence for the introduction of pulse oximetry as a screening test for critical congenital heart disease defects. No official initiatives have been presented in Colombia to generalise the practice.

(c) 2018 Sociedad Colombiana de Cardiología y Cirugía Cardiovascular. Published by Elsevier España, S.L.U. This is an open access article under the CC BY-NC-ND license (http:// creativecommons.org/licenses/by-nc-nd/4.0/).
\end{abstract}

\section{Introducción}

Los defectos cardiacos congénitos o defectos cardiacos congénitos son la malformación congénita más frecuente, con incidencias de 6 a 9 por 1.000 nacidos vivos ${ }^{1-4}$ y suelen presentarse como hallazgo aislado; sin embargo, pueden ocurrir en el contexto de cromosomopatías, síndromes monogénicos o exposición a teratógenos ${ }^{5}$.

Los defectos cardiacos congénitos ocasionan 6 al 10\% de las muertes infantiles, 20 al $40 \%$ de las muertes por malformaciones y $30 \%$ de las muertes prenatales ${ }^{7}$, representando más muertes que cualquier otro tipo de malformación y constituyendo un reto médico y social ${ }^{6-10}$.

El $25 \%$ de los casos de recién nacidos vivos con defectos cardiacos congénitos son críticos ${ }^{11,12}$. Estas cardiopatías congénitas críticas o cardiopatías congénitas críticas pueden ser tratadas con cirugía o cateterismo, y su reconocimiento temprano es crucial para un buen resultado, pero es difícil debido a la falta de signos clínicos específicos al inicio del periodo neonatal ${ }^{1,13}$.

Una proporción significativa de recién nacidos con cardiopatías congénitas críticas no se diagnostica antes del alta $^{14,15}$, lo cual podría explicarse en parte por la tendencia de los médicos a una práctica en la que a la valoración clínica no se le da la importancia que le corresponde y por tanto no se realizan exámenes físicos cardiovasculares detallados, pero también por tendencias recientes de alta hospitalaria temprana ${ }^{16}$.

El ductus arteriosus puede permanecer patente por más de 72 horas, incluso en recién nacidos a término ${ }^{17}$, de ahí que 20 a $25 \%$ de los recién nacidos con cardiopatías congénitas críticas puede salir de la sala de maternidad sin el diagnóstico ${ }^{15,16,18}$, hallazgo que ilustra la sensibilidad insuficiente del examen clínico para detectar las cardiopatías congénitas críticas ${ }^{1}$.

Por otro lado, la tamización neonatal ha llevado a mejoras dramáticas en la morbimortalidad en los lugares donde se ha implementado ${ }^{19}$. Históricamente, la tamización neonatal se ha basado en el análisis de manchas secas de sangre y ha operado como un trabajo conjunto entre los profesionales de la salud y los sistemas de salud pública ${ }^{20}$. En los últimos años, la pulsioximetría ha venido consolidándose como una alternativa clave y efectiva para la implementación de la tamización neonatal de cardiopatías congénitas críticas.

\section{Cardiopatías congénitas críticas}

Son un grupo de desórdenes morfológicamente heterogéneos, los cuales tienen en común el requerimiento de cirugía temprana o terapia intervencionista con catéter para alcanzar la supervivencia ${ }^{21-23}$.

La mayoría de los recién nacidos con cardiopatías congénitas críticas pueden ser diagnosticados con ecocardiografía, estabilizados con infusión de prostaglandina y tratados con cirugía o cateterismo ${ }^{11}$. Sin embargo, el examen clínico no detecta con prontitud todas las formas de cardiopatías congénitas críticas ${ }^{24,25}$, a riesgo de que su primera manifestación sea un colapso circulatorio, de manera que el diagnóstico tardío está asociado con una morbimortalidad significativa ${ }^{13,23,26-28}$. Además, un estado clínico deficiente al momento de la cirugía aumenta la mortalidad $^{13}$ y por el contrario, el diagnóstico oportuno mejora el resultado ${ }^{29-31}$. 
La incidencia actual de compromiso fisiológico severo por una cardiopatía congénita crítica no reconocida se ha estimado en 1 por 15.000 a 1 por 26.000 nacidos vivos ${ }^{32}$.

\section{Tamización de cardiopatías congénitas críticas}

Un subtipo de cardiopatías congénitas críticas son las dependientes de ductus; estas son cardiopatías congénitas críticas clínicamente reconocibles sólo después del cierre espontáneo del ductus arteriosus $s^{1,33}$, cuando se produce disminución severa de la eyección cardiaca, cianosis, deterioro rápido del paciente, necesidad de ventilación prolongada y alta mortalidad $^{1,13}$. De 1 a 1,8 por cada 1.000 nacidos vivos tienen una circulación dependiente del ductus, considerándose primordial la persistencia del ductus arteriosus para la supervivencia de aquellas con obstrucciones severas al flujo sistémico y disminución aguda de la eyección cardiaca ${ }^{15,34,35}$, lo cual evidencia la importancia de su detección oportuna, antes del cierre del ductus ${ }^{1}$.

Los primeros trabajos que examinaron la pulsioximetría como una herramienta de tamización para circulaciones dependientes del ductus fueron publicados en los 90 , pero tenían tamaños de muestra insuficientes para la determinación de la sensibilidad y especificidad ${ }^{6,7,34-40}$. Entre 2008 y 2010, se dieron a conocer los resultados de estudios grandes que mostraron sensibilidades considerables, altas especificidades y bajas tasas de falsos positivos, y de esta manera se fue confirmando la idoneidad de la pulsioximetría como herramienta para la tamización neonatal de circulaciones dependientes del ductus y/o cardiopatías que producen hipoxemia; además, estos estudios fueron ofreciendo hallazgos específicos sobre los puntos de corte y otros aspectos de importancia en la consolidación de la pulsioximetría como herramienta de tamización, como por ejemplo que el tipo de pulsioxímetro influye en los resultados, que la pulsioximetría tanto pre- como postductal permite detectar más casos y que la implementación de la tamización neonatal con pulsioximetría facilita la detección de pacientes con hipoxia por enfermedades diferentes a las cardiopatías congénitas ${ }^{21,41-43}$. En la tabla 1 se describen los principales hallazgos de los estudios con mayor tamaño de muestra realizados al respecto.

\section{El abordaje estadounidense, 2011}

En Estados Unidos, antes del año 2010, la única condición no incluida en el paradigma de la mancha seca de sangre para la que se recomendaba tamización neonatal era la hipoacusia ${ }^{20,44-46}$.

Basándose en una revisión exhaustiva de la evidencia, que incluyó una revisión hecha en 2009 por la AAP (American Academy of Pediatrics) y la AHA (American Heart Association) donde se encontraron razones de peso para realizar tamización con pulsioximetría ${ }^{20}$, al igual que en los estudios sueco $^{47}$ y alemán ${ }^{21}$ (tabla 1 ), entre otros, en septiembre de 2010 la Secretaría del Comité de Consejería en Enfermedades Hereditarias en Recién Nacidos y Niños de Estados Unidos (SACHDNC su sigla en inglés), recomendó que la enfermedad cardiaca cianótica congénita fuera adicionada al panel de tamización ${ }^{20}$. La SACHDNC consideró siete lesiones específicas como objetivos primarios para tamización: síndrome de corazón izquierdo hipoplásico, atresia pulmonar, tetralogía de Fallot, retorno venoso pulmonar anómalo total, trasposición de grandes arterias, atresia tricuspídea y tronco arterioso. Este subconjunto de lesiones excluía aquellas que usualmente no se asocian con hipoxia, como la estenosis valvular aórtica ${ }^{11}$.

La SACHDNC, en colaboración con la AAP, la AHA y la ACCF (American College of Cardiology Foundation) desarrollaron la estrategia de implementación y cambiaron el término previo para referirse a las siete condiciones ("enfermedad cardiaca cianótica congénita") por un nuevo término: "enfermedades cardiacas congénitas críticas", debido a que muchos de los recién nacidos con las condiciones objetivo no desarrollan cianosis clínicamente apreciable hasta después del egreso, y a que algunas lesiones, como el síndrome de corazón izquierdo hipoplásico, pueden manifestarse con compromiso cardiovascular significativo sin cianosis aparente.

La metodología para el desarrollo de dicha estrategia consistió en invitar a un grupo de trabajo a un encuentro de dos días con el objetivo de fijar recomendaciones para la tamización con pulsioximetría para la detección de cardiopatías congénitas críticas. El grupo se enfocó en la tamización en recién nacidos aparentemente sanos y aquellos con estancias cortas en las salas de cuidado intermedio. Se recomendó el uso de pulsioxímetros tolerantes al movimiento que reportan la saturación de oxígeno funcional, validados en condiciones de baja perfusión, autorizados por la FDA para el uso en recién nacidos y con alta precisión. Dado que una tamización muy temprana puede llevar a falsos positivos, a causa de la transición de la circulación fetal a la neonatal y la estabilización de los niveles de saturación de oxígeno, y una tamización tardía puede implicar que se pierda la oportunidad de intervenir antes de que se cierre el ductus arteriosus, también se recomendó que la tamización no se hiciera antes de las primeras 24 horas de vida, o que se realizara tan tarde como fuera posible si se planeaba dar una salida temprana y que esta se repitiera en el segundo día de vida. Igualmente se recomendó que la tamización se efectuara en la mano derecha (circulación preductal) y en uno de los dos pies (circulación postductal).

Se estableció que un resultado de tamización debe ser considerado positivo (en localidades ubicadas a nivel del mar) si se cumple una de las siguientes tres condiciones:

1. Cualquiera de las dos medidas de saturación dan un resultado menor de $90 \%$.

2. La saturación de oxígeno es menor de $95 \%$ en ambas extremidades, pero este resultado debe repetirse tres veces con una separación de una hora.

3. Cuando haya una diferencia absoluta de más de $3 \%$ entre las saturaciones de la mano derecha y uno de los dos pies.

Un hecho de gran relevancia es que el grupo de trabajo también enfatizó en la importancia de no reemplazar el examen físico completo ni la historia clínica por la pulsioximetría, ya que a través de estos a veces pueden detectar cardiopatías congénitas críticas antes del desarrollo de hipoxemia. Se determinó que cada recién nacido con un resultado positivo en la tamización requiere inicialmente una evaluación de las causas de la hipoxemia. 
Tabla 1 Cronología de los hallazgos en la investigación sobre la estrategia de tamizaje con pulsioximetría

\begin{tabular}{|c|c|c|c|c|c|c|}
\hline Estudio & $\begin{array}{l}\text { Estudio en Noruega, } \\
2008 \text { (41) }\end{array}$ & $\begin{array}{l}\text { Estudio sueco, } 2009 \\
\text { (42) }\end{array}$ & $\begin{array}{l}\text { Estudio alemán, } 2010 \\
\text { (21) }\end{array}$ & $\begin{array}{l}\text { Abordaje en EUA, } \\
2011 \text { (43) }\end{array}$ & $\begin{array}{l}\text { Metaanálisis, } 2012 \\
\text { (23) }\end{array}$ & $\begin{array}{l}\text { Estudio en China, } \\
2014 \text { (49) }\end{array}$ \\
\hline Objetivos y dianas & $\begin{array}{l}\text { Estimar el desempeño } \\
\text { de la pulsioximetría } \\
\text { en la detección de } \\
\text { trastornos con } \\
\text { saturación arterial de } \\
\text { oxígeno inferior a lo } \\
\text { normal, } \\
\text { especialmente } \\
\text { defectos cardíacos } \\
\text { congénitos. }\end{array}$ & $\begin{array}{l}\text { Estimar el desempeño } \\
\text { de la pulsioximetría } \\
\text { en la detección de } \\
\text { enfermedad cardíaca } \\
\text { congénita, } \\
\text { específicamente } \\
\text { circulación } \\
\text { dependiente del } \\
\text { ductus. }\end{array}$ & $\begin{array}{l}\text { Estimar el desempeño } \\
\text { de la pulsioximetría } \\
\text { en la detección } \\
\text { de cardiopatías } \\
\text { congénitas críticas: } \\
\text { Circulación sistémica } \\
\text { dependiente del } \\
\text { ductus, circulación } \\
\text { pulmonar } \\
\text { dependiente del } \\
\text { ductus, drenaje } \\
\text { venoso pulmonar } \\
\text { anómalo total, } \\
\text { transposición de las } \\
\text { grandes arterias, } \\
\text { enfermedad cardíaca } \\
\text { congénita cianótica. }\end{array}$ & $\begin{array}{l}\text { Generar un algoritmo } \\
\text { para la } \\
\text { implementación de la } \\
\text { pulsioximetría en la } \\
\text { tamización neonatal } \\
\text { para la detección } \\
\text { de "Enfermedad } \\
\text { cardiaca congénita } \\
\text { crítica"' en recién } \\
\text { nacidos } \\
\text { aparentemente } \\
\text { sanos. }\end{array}$ & $\begin{array}{l}\text { Estimar el desempeño } \\
\text { de la pulsioximetría } \\
\text { en la detección } \\
\text { de cardiopatías } \\
\text { congénitas críticas } \\
\text { en recién nacidos } \\
\text { asintomáticos. }\end{array}$ & $\begin{array}{l}\text { Estimar el desempeño } \\
\text { de la pulsioximetría } \\
\text { en la detección } \\
\text { de cardiopatías } \\
\text { congénitas mayores: }\end{array}$ \\
\hline Tamaño de muestra & 50.008 & 39.821 & 41.442 & - & 229.421 & 122.738 \\
\hline Valores de corte & $\begin{array}{l}\mathrm{SO}_{2} \text { postductal }<95 \% \\
\text { en el primer día de } \\
\text { vida. }\end{array}$ & $\begin{array}{l}\mathrm{SO}_{2}<95 \% \text {, diferencia } \\
\text { pre-postductal }>3 \%\end{array}$ & $\begin{array}{l}\mathrm{SpO}_{2} \leq 95 \% \text { en } \\
\text { cualquiera de los } \\
\text { pies, y confirmado } \\
\text { después de } 1 \text { hora. }\end{array}$ & $\begin{array}{l}\mathrm{SO}_{2} \text { preductal o } \\
\text { postductal< }<0 \%, \mathrm{SO}_{2} \\
\text { preductal y } \\
\text { postductal< }<95 \% \\
\text { diferencia absoluta } \\
\text { de }>3 \% \text { (confirmado } \\
\text { tres veces y } \\
\text { separadas por una } \\
\text { hora). }\end{array}$ & - & $\begin{array}{l}\mathrm{SO}_{2} \text { preductal o } \\
\text { postductal }<90 \%, \mathrm{SO}_{2} \\
\text { preductal y } \\
\text { postductal }<95 \% ; \\
\text { diferencia absoluta } \\
\text { de }>3 \% \text { (confirmado } \\
\text { tres veces y } \\
\text { separadas por una } \\
\text { hora). }\end{array}$ \\
\hline $\begin{array}{l}\text { Confirmación } \\
\text { de casos }\end{array}$ & Ecocardiografía & Ecocardiografía & $\begin{array}{l}\text { Examen clínico } \\
\text { completo y } \\
\text { ecocardiografía }\end{array}$ & Ecocardiografía & - & $\begin{array}{l}\text { Ecocardiografía, } \\
\text { seguimiento clínico } \\
\text { y retroalimentación } \\
\text { de padres }\end{array}$ \\
\hline
\end{tabular}




\begin{tabular}{|c|c|c|c|c|c|c|}
\hline Estudio & $\begin{array}{l}\text { Estudio en Noruega, } \\
2008 \text { (41) }\end{array}$ & $\begin{array}{l}\text { Estudio sueco, } 2009 \\
(42)\end{array}$ & $\begin{array}{l}\text { Estudio alemán, } 2010 \\
(21)\end{array}$ & $\begin{array}{l}\text { Abordaje en EUA, } \\
2011(43)\end{array}$ & $\begin{array}{l}\text { Metaanálisis, } 2012 \\
(23)\end{array}$ & $\begin{array}{l}\text { Estudio en China, } \\
2014(49)\end{array}$ \\
\hline Sensibilidad & $\begin{array}{l}\text { Defectos cardiacos } \\
\text { congénitos: } 9,9 \% \\
(7,3-13,2) \\
\text { Cardiopatías } \\
\text { congénitas críticas: } \\
77,1 \%(59,4-89,0)\end{array}$ & $62,07 \%(42,3-79,31)$ & $77,8 \%$ & - & $76,5 \%(67,7-83,5)$ & $\begin{array}{l}\text { Cardiopatías } \\
\text { congénitas mayores: } \\
58,7 \%(53,2-64,0) \\
\text { Cardiopatías } \\
\text { congénitas críticas: } \\
83,6 \%(76,7-88,7)\end{array}$ \\
\hline Especificidad & $\begin{array}{l}\text { Defectos cardiacos } \\
\text { congénitos: } 99,4 \% \\
(99,3-99,5) \\
\text { Cardiopatías } \\
\text { congénitas } \\
\text { críticas:99,4\% } \\
(99,3-99,5)\end{array}$ & $99,82 \%(99,77-99,86)$ & $99,90 \%$ & - & $99,9 \%(99,7-99,9)$ & $\begin{array}{l}\text { Cardiopatías } \\
\text { congénitas mayores: } \\
99,7 \%(99,7-99,8) \\
\text { Cardiopatías } \\
\text { congénitas críticas: } \\
99,7 \%(99,6-99,7)\end{array}$ \\
\hline $\begin{array}{c}\text { Tasa de falsos } \\
\text { positivos }\end{array}$ & $\begin{array}{l}\text { Defectos cardiacos } \\
\text { congénitos: } 0,6 \% \\
(0,5-0,6) \\
\text { Cardiopatías } \\
\text { congénitas críticas: } \\
0,6 \%(0,5-0,7)\end{array}$ & $0,17 \%$ & $0,09 \%$ & - & $0,14 \%(0,06-0,33)$ & $\begin{array}{l}\text { Cardiopatías } \\
\text { congénitas mayores: } \\
0,3 \% \\
\text { Cardiopatías } \\
\text { congénitas críticas: } \\
0,3 \%\end{array}$ \\
\hline
\end{tabular}


En ausencia de otros hallazgos que expliquen la hipoxemia, deberá realizarse ecocardiograma para descartar cardiopatías congénitas críticas ${ }^{20}$.

\section{Un metaanálisis en 2012}

En 2012 se publicó un metaanálisis realizado con base en estudios planteados hasta el año 2010. Se determinó como objetivo la evaluación del comportamiento de la pulsioximetría como un método de tamización para la detección de defectos cardíacos congénitos críticos en recién nacidos asintomáticos. Se seleccionaron estudios que incluían recién nacidos asintomáticos tamizados con pulsioximetría, considerando afectado por defecto cardíaco congénito crítico a todo infante que moría o necesitaba cirugía en los primeros 28 días de vida. Mediante la revisión sistemática de bibliografía relevante de 552 estudios, se identificaron 13 estudios primarios como apropiados para su inclusión, con datos de 229.421 recién nacidos. Los hallazgos obtenidos demostraron que la pulsioximetría es un test de alta especificidad $(99,9 \%)$, sensibilidad moderada $(76,5 \%)$ y bajas tasas de resultados falsos positivos para la detección de defectos cardíacos congénitos críticos en recién nacidos asintomáticos (tabla 1). Las tasas de falsos positivos se vieron afectadas por el tiempo de la prueba y fueron significativamente más bajas cuando la tamización se realizó 24 horas después del nacimiento. No se notaron diferencias significativas en la sensibilidad para la pulsioximetría cuando se obtuvieron las muestras solo en el pie; sin embargo, se resalta que en estudios que usaron ambas saturaciones, preductal y postductal, se detectaron defectos individuales que no habrían sido identificados por saturaciones postductales solas. Este artículo recopiló el más amplio conjunto de datos de precisión hasta ese momento. Los hallazgos de este metaanálisis proveen evidencia decisiva para la introducción de la pulsioximetría como un método de tamización en la práctica clínica ${ }^{23}$.

\section{Un estudio en la unidad de cuidado intensivo neonatal, 2014}

Debido a que el protocolo propuesto por el grupo de trabajo convocado por SACHDNC para tamización de recién nacidos es recomendado sólo en salas de recién nacidos aparentemente sanos y de cuidados intermedios ${ }^{20}$, en el año 2014 se convocó un grupo de trabajo con el objetivo de analizar la implementación de la tamización por pulsioximetría en Unidades de Cuidado Intensivo Neonatal (UCIN). Se realizó un estudio utilizando el protocolo de la SACHDNC destinado a recién nacidos en aparente buen estado sin ninguna modificación, excepto que la tamización se hacía al momento de dar de alta de la UCIN a los neonatos. Se consideraron 500 admisiones a una UCIN académica de tercer nivel, 250 admisiones consecutivas antes de que se implementara el protocolo de tamización, de noviembre 7 a diciembre 31 de 2011 (grupo 1) y 250 admisiones consecutivas después de la implementación del protocolo, de agosto 1 a octubre 9 de 2012 (grupo 2). Todos los recién nacidos, excepto aquellos que tuvieron un ecocardiograma completo durante su estadía en el hospital, fueron sometidos a tamización por pulsioximetría antes de darles de alta. Los resultados del estudio mostraron que en ausencia de un protocolo en la unidad, aproximadamente el $5 \%$ de todos los pacientes de la UCIN fueron dados de alta sin pulsioximetría durante su estadía o con una medición de $\mathrm{SpO}_{2}<95 \%$ en el día que se les dio de alta. Los resultados del estudio muestran que infantes pretérmino tardío y a término que requirieron admisión en la UCIN, al darles de alta tuvieron saturaciones de oxígeno similares a las de los infantes sin morbilidades. También confirmaron que las saturaciones de oxígeno preductal y postductal en la mayoría de los infantes pretérmino al ser dados de alta, no son diferentes de las de los infantes pretérmino tardío y a término. En conclusión, se determinó que el protocolo recomendado para el uso en infantes en salas de recién nacidos en aparente buen estado y cuidado intermedio, puede ser usado con infantes dados de alta desde $U_{C I N}{ }^{48}$ (tabla 1).

\section{El estudio Chino, 2014}

Habiéndose demostrado la efectividad de la pulsioximetría como herramienta de tamización neonatal de cardiopatías congénitas críticas en países de altos ingresos, investigadores chinos realizaron un estudio prospectivo y multicéntrico en su país para evaluar su utilidad, la de la evaluación clínica, o ambas, en la tamización de cardiopatías congénitas críticas en 122.738 recién nacidos sin evidencia de defectos cardiacos congénitos en la ecografía prenatal. La pulsioximetría fue realizada por un clínico entre las 6-72 horas de vida e inmediatamente después este mismo efectuó la evaluación clínica. Se usó el protocolo propuesto por la Secretaría de Salud y Servicios Humanos de los Estados Unidos. En comparación con la evaluación clínica, la pulsioximetría tuvo mayor especificidad $(99,7 \%$ vs. $97,3 \%)$, mayor sensibilidad $(83,6 \%$ vs. $77,4 \%)$, y menor tasa de falsos positivos $(0,3 \%$ vs. $2,7 \%)$. Para la detección de todo tipo de cardiopatías, la combinación de pulsioximetría más evaluación clínica fue mejor que cada uno de los métodos por separado. Este es el estudio de evaluación de la precisión de esta herramienta de tamización que tiene mayor tamaño de muestra, y el primero que refleja el desempeño de esta intervención en un país de bajos ingresos. Sus resultados proveen un argumento fuerte para la implementación de la tamización de cardiopatías congénitas críticas por pulsioximetría en países de bajos ingresos ${ }^{49}$ (tabla 1).

\section{Situación en Colombia}

En la actualidad, los datos estadísticos que se tienen sobre la incidencia de defectos congénitos en Colombia son escasos; aun así, se sabe que por ejemplo para el año 2012, de todos los defectos congénitos notificados al Instituto Nacional de Salud, el $19 \%$ fueron cardiopatías congénitas, lo cual evidencia su importancia ${ }^{50}$ y está en congruencia con las cifras presentadas para los países de mediano y bajo ingreso económico $(25 \%)$ respecto a defectos congénitos encabezados por las cardiopatías congénitas.

En 2013 se publicó en Colombia la Guía de Práctica Clínica del Sistema General de Seguridad Social en Salud para la Detección de Anomalías Congénitas en el Recién Nacido, 
Tabla 2 Criterios clásicos de tamización de enfermedades (Wilson y Jungner, 1968)

Criterio
problema de salud importante
Debe existir un tratamiento aceptado
para los pacientes con enfermedad
reconocida

Las instalaciones para el diagnóstico y tratamiento deben estar disponibles

Debe existir una etapa de latencia o sintomática temprana reconocible

Debe existir una prueba o examen adecuado

La prueba debe ser aceptable para la población

La historia natural de la condición, desde su periodo latente hasta la enfermedad declarada, debe ser bien conocida.

Debe existir una política consenso acerca de quién tratar como paciente

$\begin{array}{ll}\text { Comentario } & \text { Referencias } \\ \text { Las incidencias estimadas para defectos } & \text { Bjornard et al., 2013; Kuelling } \\ \text { cardiacos congénitos son } 6 \text { a } 9 \text { por } & \text { et al., 2009; Schultz et al., } \\ 1.000 \text { nacidos vivos, y la incidencia actual } & \text { 2008; Yang et al., 2006. }\end{array}$

de compromiso fisiológico severo por cardiopatía congénita crítica no reconocida se ha estimado en 1 por 15.000 a 1 por 26.000 nacidos vivos.

Las cardiopatías congénitas críticas son un grupo de desórdenes morfológicamente heterogéneos, los cuales tienen en común el requerimiento de cirugía temprana o terapia intervencionista con catéter para alcanzar la supervivencia.

Existen varios centros de referencia para el diagnóstico y tratamiento de patologías cardíacas distribuidos a lo largo y ancho del país. En las zonas específicas donde estos se encuentran y en general en sus áreas de influencia, este criterio se cumple. Sin embargo, existen zonas del país que tendrían dificultad para acceder oportunamente a un tratamiento adecuado. En países que comparten esta problemática, una solución efectiva ha sido el establecimiento de redes de atención que implican el uso de telemedicina; que exigen compromiso y esfuerzo de las autoridades de salud.

Cerca del $50 \%$ de los niños con cardiopatías congénitas críticas no hallazgos al examen físico incluyendo soplo durante las primeras horas de vida, y la manifestación inicial después de este periodo asintomático, puede ser el colapso cardiovascular. Múltiples estudios descritos en esta revisión, incluido un estudio en países en desarrollo, demuestran la idoneidad de la pulsioximetría como herramienta para detección de cardiopatías congénitas críticas.

La pulsioximetría es un procedimiento rutinario no invasivo, fácilmente aceptado por los padres y por el personal de salud. La fisiopatología e historia natural de las cardiopatías congénitas críticas es bien conocida.

Las cardiopatías congénitas críticas son un grupo de desórdenes morfológicamente heterogéneos, los cuales tienen en común el requerimiento de una cirugía temprana o terapia intervencionista con catéter alcanzar la supervivencia.
Liberman et al., 2014; Riede et al., 2010; Thangaratinam et al., 2012.

Al Mazrouei et al., 2013.

Brown et al., 2006; Kuelling et al., 2009.

Kemper et al.,. 2011; Riede et al., 2010; Thangaratinam et al., 2012. US Preventive Services Task Force, 2008. González, 2012.

Al Mazrouei et al., 2013.

Brown et al., 2006; Kuelling et al., 2009.

Liberman et al., 2014; Riede et al., 2010; Thangaratinam et al., 2012. 
Tabla 2 (continuación)

\begin{tabular}{|c|c|c|}
\hline Criterio & Comentario & Referencias \\
\hline $\begin{array}{l}\text { El costo del hallazgo de los casos debe } \\
\text { estar económicamente equilibrado } \\
\text { en relación con el gasto total de la } \\
\text { asistencia médica }\end{array}$ & $\begin{array}{l}\text { Estudios en Estados Unidos y Europa } \\
\text { muestran que la tamización con } \\
\text { pulsioximetría es una intervención } \\
\text { costo-efectiva; se ha sugerido que es } \\
\text { probablemente más costo-efectiva en } \\
\text { regiones con tasas elevadas de diagnóstico } \\
\text { tardío como en los países de bajos ingresos. }\end{array}$ & $\begin{array}{l}\text { de-Wahl Granelli et al., 2009; } \\
\text { Kumar, } 2016 .\end{array}$ \\
\hline $\begin{array}{l}\text { La búsqueda de los casos debe ser un } \\
\text { proceso continuo y no un proyecto } \\
\text { "de vez en cuando", }\end{array}$ & $\begin{array}{l}\text { Existen varias localidades en el mundo } \\
\text { donde se ha implementado como un } \\
\text { mandato este método de tamización. }\end{array}$ & Kumar, 2016. \\
\hline
\end{tabular}

en la que se establece la realización de pulsioximetría postductal (en uno de los pies) después de las 24 horas de vida, seguida de ecocardiograma si esta resulta anormal. Sin embargo, este método de tamización solo se aplica en algunas instituciones en Colombia, y no han existido iniciativas estatales encaminadas a posibilitar su generalización ni hay publicaciones sobre el cumplimiento de esta recomendación.

\section{Conclusión}

Las cardiopatías congénitas son uno de los defectos congénitos más comunes y una causa principal de mortalidad infantil, hecho que las convierte en un problema de salud pública, el cual es el primero de los diez criterios de Wilson y Jungner, que son las pautas para considerar que un desorden califica para ser incluido en el panel de tamizaje neonatal, y actualmente las cardiopatías congénitas cumplen con la totalidad de ellos (tabla 2).

La tamización con pulsioximetría para la detección de cardiopatías congénitas críticas ya ha sido implementada en Estados Unidos y algunos países europeos, con base en los resultados de diferentes estudios hechos en países de altos ingresos $20,21,23,44,50$. Adicionalmente, se publicó un estudio realizado en China que mostró la factibilidad y utilidad de implementación de esta intervención en un país en vía de desarrollo.

La literatura al respecto ha mostrado el beneficio que produce la implementación de la pulsioximetría como método de tamizaje y la discusión se está dirigiendo hacia el establecimiento de protocolos ajustados a los recursos y requerimientos de cada localidad. Sin embargo, se debe enfatizar que la pulsioximetría no reemplaza el examen físico completo ni la historia clínica, ya que estos a veces pueden detectar cardiopatías congénitas críticas antes del desarrollo de hipoxemia.

Pese a que existe evidencia fuerte en la literatura mundial para establecer que los sistemas de salud consideren la introducción de la pulsioximetría como una prueba de tamización para defectos cardiacos congénitos críticos en recién nacidos asintomáticos, en Colombia no se han presentado iniciativas oficiales para generalizar la práctica.

\section{Responsabilidades éticas}

Protección de personas y animales. Los autores declaran que para esta investigación no se han realizado experimentos en seres humanos ni en animales.

Confidencialidad de los datos. Los autores declaran que en este artículo no aparecen datos de pacientes.

Derecho a la privacidad y consentimiento informado. Los autores declaran que en este artículo no aparecen datos de pacientes.

\section{Financiación}

Ninguna.

\section{Conflicto de intereses}

Ninguno.

\section{Bibliografía}

1. Kuelling B, Mieth R, Bauersfeld U, Balmer C, R AM., Bauersfeld $\mathrm{U}$, et al. Pulse oximetry screening for congenital heart defects in Switzerland: Most but not all maternity units screen their neonates. Swiss Med Wkly. 2009;139(47-48):699-704.

2. Bjornard K, Riehle-Colarusso T, Gilboa SM, Correa A. Patterns in the prevalence of congenital heart defects, metropolitan Atlanta, 1978 to 2005. In: Birth Defects Research Part A: Clinical and Molecular Teratology. 2013:87-94.

3. Yang $\mathrm{Q}$, Chen H, Correa A, Devine O, Mathews TJ, Honein MA. Racial differences in infant mortality attributable to birth defects in the United States, 1989-2002. In: Birth Defects Research Part A: Clinical and Molecular Teratology. 2006:706-13.

4. Engel MS, Kochilas LK. Pulse oximetry screening: A review of diagnosing critical congenital heart disease in newborns. Medical Devices: Evidence and Research. Dove Medical Press Ltd; 2016. p. 199-203.

5. Firth HV, Hurst JA. Oxford Desk Reference Clinical Genetics. 2005.

6. de Wahl Granelli A, Mellander M, Sunnegårdh J, Sandberg K, Ostman-Smith I. Screening for duct-dependant congenital heart 
disease with pulse oximetry: a critical evaluation of strategies to maximize sensitivity. Acta Paediatr. 2005;94:1590-6.

7. Hoffman J. Incidence of congenital heart disease: II. Prenatal incidence. Pediatr Cardiol. 1995;16:155-6.

8. Knowles R, Griebsch I, Dezateux C, Brown J, Bull C, Wren C. Newborn screening for congenital heart defects: a systematic review and cost-effectiveness analysis. Health Technol Assess. 2005;9:1-152.

9. Lloyd J, Askie L, Smith J, Tarnow-Mordi W. Supplemental oxygen for the treatment of prethreshold retinopathy of prematurity. Cochrane Database Syst Rev. 2003:CD003482.

10. Lin C-J, Lin C-Y, Chen C-H, Zhou B, Chang C-P. Partitioning the heart: mechanisms of cardiac septation and valve development. Development [Internet]. 2012;139:3277-99 [cited 2014 Dec 9] Disponible en: http://www.pubmedcentral. nih.gov / articlerender.fcgi? artid = 3424040\&tool = pmcentrez\& rendertype $=$ Abstract.

11. Mahle WT, Newburger JW, Matherne GP, Smith FC, Hoke TR, Koppel $\mathrm{R}$, et al. Role of pulse oximetry in examining newborns for congenital heart disease: a scientific statement from the American Heart Association and American Academy of Pediatrics. Circulation [Internet]. 2009;120:447-58 [cited 2014 Dec 11] Disponible en: http://www.ncbi.nlm.nih.gov/pubmed/1958149212.

12. Bruno CJ, Havranek T. Screening for Critical Congenital Heart Disease in Newborns. Advances in Pediatrics. 2015.

13. Brown KL, Ridout Da, Hoskote A, Verhulst L, Ricci M, Bull C. Delayed diagnosis of congenital heart disease worsens preoperative condition and outcome of surgery in neonates. Heart [Internet]. 2006;92:1298-302 [cited 2014 Dec 29] Disponible en: http://www.pubmedcentral.nih.gov/articlerender.fcgi?artid = 1861169\&tool = pmcentrez\&rendertype = Abstract.

14. Chang R, Rodriguez S, Klitzner T. Screening newborns for congenital heart disease with pulse oximetry: survey of pediatric cardiologists. Pediatr Cardiol. 2009;30:20-5.

15. Wren C, Reinhardt Z, Khawaja K. Twenty-year trends in diagnosis of life-threatening neonatal cardiovascular malformations. Arch Dis Child Fetal Neonatal Ed. 2008;93:F33-5.

16. Mellander M, Sunnegårdh J. Failure to diagnose critical heart malformations in newborns before discharge-an increasing problem? Acta Paediatr. 2006;95:407-13.

17. Alenick DS, Holzman IR, Ritter SB. The neonatal transitional circulation: a combined noninvasive assessment. Echocardiography. 1992;9:29-37.

18. Abu-Harb M, Hey E, Wren C. Death in infancy from unrecognised congenital heart disease. Arch Dis Child [Internet]. 1994;71:3-7. Disponible en: http://adc.bmj.com/ cgi/doi/10.1136/adc.71.1.3.

19. Therell B, Lorey F, Eaton R. Impact of expanded newborn screening-United States, 2006. MMWR Morb Mortal Wkly Rep. 2008; $57: 1012-5$

20. Kemper AR, Mahle WT, Martin GR, Cooley WC, Kumar P, Morrow WR, et al. Strategies for implementing screening for critical congenital heart disease. Pediatrics. 2011;128:e1259-67.

21. Riede FT, Wörner C, Dähnert I, Möckel A, Kostelka M, Schneider P. Effectiveness of neonatal pulse oximetry screening for detection of critical congenital heart disease in daily clinical routine-results from a prospective multicenter study. Eur J Pediatr [Internet]. 2010;169:975-81 [cited 2014 Dec 22] Disponible en: http://www.pubmedcentral.nih.gov/ articlerender . cgi artid $=2890074 \&$ tool $=$ pmcentrez\& rendertype $=$ Abstract.

22. Liberman RF, Getz KD, Lin AE, Higgins CA, Sekhavat S, Markenson GR, et al. Delayed diagnosis of critical congenital heart defects: trends and associated factors. Pediatrics [Internet]. 2014;134:e373-81 [cited 2014 Dec 22] Disponible en: http://www.ncbi.nlm.nih.gov/pubmed/2507030123.

23. Thangaratinam S, Brown K, Zamora J, Khan KS, Ewer AK. Pulse oximetry screening for critical congenital heart defects in asymptomatic newborn babies: a systematic review and meta-analysis. Lancet [Internet], 379. Elsevier Ltd; 2012. p. 2459-64 [cited 2014 Dec 5] Disponible en: http://www.ncbi.nlm.nih.gov/pubmed/22554860.

24. Ainsworth SB, Wyllie JP, Wren C. Prevalence and clinical significance of cardiac murmurs in neonates. Arch Dis Child Fetal Neonatal Ed [Internet]. 1999;80:F43-5. Disponible en: http://fn.bmj.com/cgi/doi/10.1136/fn.80.1.F43.

25. Wren C, Richmond S, Donaldson L, Royal S. Presentation of congenital heart disease in infancy?: implications for routine examination. Arch Dis Child Fetal Neonatal Ed. 1999;80:F49-53.

26. Chang R-KR, Gurvitz M, Rodriguez S. Missed diagnosis of critical congenital heart disease. Arch Pediatr Adolesc Med [Internet]. 2008;162:969-74. Disponible en: http: / / www.ncbi.nlm.nih.gov/pubmed/18838650.

27. Kuehl K, Loffredo C, Ferencz C. Failure to diagnose congenital heart disease in infancy. Pediatrics. 1999;103 4 Pt 1:743-7.

28. Malkin J, Garber S, Broder M, Keeler E. Infant mortality and early postpartum discharge. Obs Gynecol. 2000;96:183-8.

29. Brown JW, Park HJ, Turrentine MW. Arterial switch operation: factors impacting survival in the current era. Ann Thorac Surg [Internet]. 2001;71:1978-84. Disponible en: http: / / linkinghub.elsevier.com/retrieve/pii/S0003497501025292.

30. Franklin O, Burch M, Manning N, Sleeman K, Gould S, Archer N, et al. Prenatal diagnosis of coarctation of the aorta improves survival and reduces morbidity. Heart. 2002;87:67-9.

31. Tworetzky W, McElhinney DB, Reddy VM, Brook MM, Hanley FL, Silverman NH. Improved surgical outcome after fetal diagnosis of hypoplastic left heart syndrome. Circulation. 2001;103:1269-73.

32. Schultz AHA, Localio AR, Clark BJB, Ravishankar C, Videon $\mathrm{N}$, Kimmel SES. Epidemiologic features of the presentation of critical congenital heart disease: implications for screening. Pediatrics. 2008;121:751-7.

33. Shah F, Chatterjee R, Patel PC, Kunkulol RR. Early detection of critical congenital heart disease in newborns using pulse oximetry screening [Internet]. International Journal of Medical Research \& Health Sciences. 2015:78-83. Disponible en: http: / /ijmrhs.com/41/13Shah et al.

34. Richmond S, Reay G, Abu Harb M. Routine pulse oximetry in the asymptomatic newborn. Arch Dis Child Fetal Neonatal Ed. 2002;87:F83-8.

35. Koppel RIR, Druschel CCM, Carter T, Goldberg BEB, Mehta PPN, Talwar R, et al. Effectiveness of pulse oximetry screening for congenital heart disease in asymptomatic newborns. Pediatrics. 2003;111:451-5.

36. Reich JD, Miller S, Brogdon B, Casatelli J, Gompf TC, Huhta JC, et al. The use of pulse oximetry to detect congenital heart disease. J Pediatr. 2003;142:268-72.

37. Hoke TR, Donohue PK, Bawa PK, Mitchell RD, Pathak A, Rowe $P C$, et al. Oxygen saturation as a screening test for critical congenital heart disease: a preliminary study. Pediatr Cardiol. 2002;23:403-9.

38. Bakr AF, Habib HS. Combining pulse oximetry and clinical examination in screening for congenital heart disease. Pediatr Cardiol. 2005;26:832-5.

39. Rosati E, Chitano G, Dipaola L, De Felice C, Latini G. Indications and limitations for a neonatal pulse oximetry screening of critical congenital heart disease. J Perinat Med. 2005;33:455-7.

40. Arlettaz R, Bauschatz AS, Mönkhoff M, Essers B, Bauersfeld U. The contribution of pulse oximetry to the early detection of congenital heart disease in newborns. Eur J Pediatr. 2006;165: 94-8.

41. Meberg A, Brügmann-Pieper S, Due R, Eskedal L, Fagerli I, Fars$\operatorname{tad} \mathrm{T}$, et al. First Day of Life Pulse Oximetry Screening to Detect Congenital Heart Defects. J Pediatr. 2008;152:761-5.

42. de-Wahl Granelli A, Wennergren M, Sandberg K, Mellander M, Bejlum C, Inganäs L, et al. Impact of pulse oximetry screening 
on the detection of duct dependent congenital heart disease: a Swedish prospective screening study in 39,821 newborns. BMJ [Internet]. 2009;338:a3037 [cited 2014 Dec 5] Disponible en: http://www.pubmedcentral.nih.gov/articlerender.fcgi?artid = 2627280\&tool $=$ pmcentrez\&rendertype $=$ Abstract .

43. Kemper AAR, William T. Strategies for Implementing Screening for Critical Congenital Heart Disease. Pediatrics. 2011;128:e1259-67.

44. US Preventive Services Task Force. Universal screening for hearing loss in newborns: US Preventive Services Task Force recommendation statement. Pediatrics. 2008;122:143-8.

45. Liu C, Farrell J, MacNeil JR, Stone S, Barfield W. Evaluating loss to follow-up in newborn hearing screening in Massachusetts. Pediatrics. 2008;121:e335-43.

46. Spivak L, Sokol H, Auerbach C, Gershkovich S. Newborn hearing screening follow-up: factors affecting hearing aid fitting by 6 months of age. Am J Audiol. 2009;18:24-33.
47. de-Wahl Granelli A, Wennergren M, Sandberg K, Mellander M, Bejlum C, Inganäs L, et al. Impact of pulse oximetry screening on the detection of duct dependent congenital heart disease: a Swedish prospective screening study in 39,821 newborns. BMJ. 2009;338:a3037.

48. lyengar $H$, Kumar $P$, Kumar P. Pulse-oximetry screening to detect critical congenital heart disease in the neonatal intensive care unit. Pediatr Cardiol [Internet]. 2014;35:406-10 [cited 2014 Dec 22] Disponible en: http://www.ncbi.nlm.nih.gov/pubmed/24026810.

49. Zhao QM, Ma XJ, Ge XL, Liu F, Yan WL, Wu L, et al. Pulse oximetry with clinical assessment to screen for congenital heart disease in neonates in China: A prospective study. Lancet [Internet], 384. Elsevier Ltd; 2014. p. 747-54. Disponible en: https://doi.org/10.1016/S0140-6736(14)60198-7.

50. González Y. Informe final del evento anomalías congénitas hasta el periodo epídemiológico 13 del año 2012. Bogotá. 2012. 